\title{
Current evidence for anesthesia management during endovascular stroke therapy: updated systematic review and meta-analysis
}

\author{
Nitin Goyal, ${ }^{1}$ Konark Malhotra, ${ }^{2}$ Muhammad F Ishfaq, ${ }_{1}^{1}$ Georgios Tsivgoulis, ${ }^{1,3}$ \\ Christopher Nickele, ${ }^{4}$ Daniel Hoit, ${ }^{4}$ Adam S Arthur, ${ }^{4}$ Andrei V Alexandrov, ${ }^{4}$ \\ Lucas Elijovich ${ }^{1,4}$
}

\begin{abstract}
- Additional material is published online only. To view please visit the journal online (http://dx.doi.org/10.1136/ neurintsurg-2018-013916).
\end{abstract}

${ }^{1}$ Department of Neurology, University of Tennessee Health Science Center, Memphis, Tennessee, USA

${ }^{2}$ Department of Neurology, West Virginia University-Charleston Division, Charleston, West Virginia, USA

${ }^{3}$ Second Department of Neurology, "Attikon University Hospital", School of Medicine, National \& Kapodistrian University of Athens, Athens, Greece

${ }^{4}$ Department of Neurosurgery, University of Tennessee Health Science Center and SemmesMurphey Clinic, Memphis, Tennessee, USA

\section{Correspondence to} Dr. Nitin Goyal, Department of Neurology, University of Tennessee Health Science Center, Memphis, TN 38163, USA; ngoyal@uthsc.edu

NG and KM contributed equally.

Received 8 March 2018 Revised 1 May 2018 Accepted 10 May 2018 Published Online First 15 June 2018

\section{ABSTRACT}

Introduction Debate continues about the optimal anesthetic management for patients undergoing endovascular treatment (ET) of acute ischemic stroke due to emergent large vessel occlusion.

Objective To compare, using current evidence, the clinical outcomes and procedural characteristics among patients undergoing general anesthesia (GA) and local or monitored anesthesia (non-GA).

Methods We performed a systematic review and metaanalysis of all available studies that involved the use of stent retrievers for ET (stentriever group). Additionally, we included studies that were published in 2015 and later, and compared the clinical outcomes among the studies using stentrievers or no stentrievers (prestentriever group). Outcome variables included functional independence (Fl; modified Rankin Scale scores of $0-2)$, symptomatic hemorrhage, mortality, procedure duration, and vascular and respiratory complications. We calculated pooled odds ratios and $95 \%$ Cls using random-effects models.

Results Sixteen studies (three randomized controlled clinical trials (RCTs) and 13 non-randomized studies) were identified comprising 5836 patients. Although non-GA was associated with higher odds of 3-month $\mathrm{FI}$ $(\mathrm{OR}=1.57 ; 95 \% \mathrm{Cl} 1.17$ to $2.10 ; \mathrm{P}=0.003)$ and lower odds of 3-month mortality $(\mathrm{OR}=0.62 ; 95 \% \mathrm{Cl} 0.47$ to $0.82 ; P=0.0006$, substantial heterogeneity was noted across included trials. Sensitivity analyses of RCTs showed that non-GA was inversely associated with $\mathrm{FI}$ $\left(\mathrm{OR}=0.55 ; 95 \% \mathrm{Cl} 0.34\right.$ to $0.89 ; \mathrm{P}=0.01 ; \mathrm{I}^{2}=15 \%$ ), while no association was noted with mortality $(O R=1.36$; $95 \% \mathrm{Cl} 0.79$ to $2.34 ; P=0.27 ; I^{2}=0 \%$ ).

Conclusion Our updated meta-analysis demonstrates favorable results with non-GA, probably owing to inclusion of non-randomized studies. Recent singlecenter RCTs indicate that GA is associated with higher odds of $\mathrm{FI}$ at 3 months, while other outcomes are similar between the two groups.

\section{INTRODUCTION}

Five recent randomized controlled clinical trials (RCTs) provide overwhelming evidence that timely endovascular therapy (ET) in patients with acute ischemic stroke (AIS) due to emergent large vessel occlusion (ELVO) is safe and improves functional outcomes. ${ }^{1-5}$ With a number needed to treat of 4 for functional independence, ${ }^{5}$ ET has become the new standard of care worldwide for treatment of patients with AIS due to ELVO. However, the choice of anesthetic during ET continues to be debated, ${ }^{6}$ and remains unsettled even after the success of landmark RCTs.

Until the emergence of state-of-the-art stentriever devices, ${ }^{7}$ general anesthesia (GA) was a preferred option among neurointerventionalists ${ }^{8}$ to minimize the potential risks of intraprocedural complications, including vessel dissection or perforation, pain and movement of the patient during intracranial catheter navigation and clot retrieval. ${ }^{9}$ However, over several years, observational studies ${ }^{10-28}$ and meta-analyses ${ }^{29} 30$ have suggested improved outcomes with local or conscious sedation (non-GA group) in comparison with GA. Additionally, GA tends to affect cerebral autoregulation and potentially could lead to worse clinical outcomes associated with extreme reductions in systemic blood pressure. Three recently conducted RCTs that directly compared anesthesia options during ET of ELVO have reported similar or more favorable outcomes in patients randomized to GA. ${ }^{31-33} 35$ Owing to this continued controversy, we performed a systematic review and meta-analysis to compare clinical and procedural outcomes with either GA or non-GA anesthesia options during ET.

\section{METHODS}

\section{Study design and literature search}

We performed a systematic review and meta-analysis of randomized and observational studies to compare outcomes in different types of anesthesia being used in patients with ELVO during ET. A computerized literature search was done of Medline and Cochrane databases from inception to December 28, 2017, with the following search terms: 'conscious sedation', 'local anesthesia', 'general anesthesia', 'acute ischemic stroke', 'outcome', 'mechanical thrombectomy', 'endovascular treatment', 'stroke', 'revascularization'. We also searched through the bibliography of the included studies to find additional studies that were not found in our initial literature search. No other search restrictions were applied. Two authors (KM and MFI) carried out the literature search and extracted data from relevant studies. We primarily included the studies that involved 
use of stent retrievers for ET (stentriever group). Additionally, we included studies that were published in 2015 and later, and compared the clinical outcomes among the studies using stentrievers or no stentrievers (pre-stentriever group). We excluded studies that were published before 2015, without any use of stentriever devices, and for which the reported outcomes were not dichotomized or compared between GA and non-GA groups.

\section{Outcome measures}

We compared the use of non-GA and GA, and their respective impact on outcome measures in patients with AIS with ELVO treated with ET. Patients were divided into two groups: a GA group if they required intubation using generalized anesthesia before or during ET, and a non-GA group if ET was performed under monitored anesthesia. Our primary outcome measures were functional independence (FI; defined as modified Rankin Scale scores of 0-2), 3-month mortality, and any intracranial hemorrhage (ICH) at 3 months. We additionally assessed symptomatic ICH (sICH), successful reperfusion (defined by Thrombolysis in Cerebral Infarction $2 \mathrm{~b} / 3$ grades and or Thrombolysis in Myocardial Infarction grades), procedure-related complications, ventilator-associated complications, procedure duration, and physiological factors, including hemodynamic instability, and patient's movement during the procedure. Procedure-related complications included vessel dissection/perforation, migration of embolus to new territory, symptomatic groin hematoma, and any kind of hemorrhage. Ventilator-associated complications were defined as pneumonia, aspiration, and challenges related to extubation. Hemodynamic instability was defined as extreme variations in maximum and minimum measurements for systolic blood pressure, diastolic blood pressure, mean arterial pressure (MAP), and heart rate. However, no cut-off values were provided for the degree of variations and the definition was not uniform in the included studies.

\section{Data extraction and quality assessment}

Two authors (MFI and KM) independently screened all the titles or abstracts, and later evaluated all the relevant articles based on the full-text reviews. For duplicate articles, we selected those with the largest sample size or the most complete information. Data from the articles were extracted by three independent authors (NG, KM, and MFI) using a standardized form. All disagreements were resolved by reaching consensus. Studies published as post hoc analysis of RCTs that compared safety and efficacy of different types of anesthesia during ET were considered as separate studies.

Cochrane risk of bias assessment was used to evaluate the potential sources of bias in the included RCTs, whereas the quality of cohort studies was assessed using the NewcastleOttawa scale. Trials that included fewer than 10 subjects, and those that did not report the outcome variables specified in our meta-analysis were excluded.

\section{Statistical analysis}

We calculated OR and 95\% CI of various variables to measure the effect size for comparison between the use of GA and non-GA during ET. We also performed adjusted analyses for efficacy outcomes for both GA and non-GA. Additionally, we performed subgroup analyses according to the study design and the type of endovascular device.

Heterogeneity was quantified using Cochran $\mathrm{Q}$ and $\mathrm{I}^{2}$ statistics according to the Cochrane Handbook. $\mathrm{I}^{2}>50 \%$ and $\mathrm{I}^{2}>75 \%$ cut-off points were used for substantial and considerable heterogeneity, respectively. We graphically evaluated the publication bias across the studies using a funnel plot, ${ }^{34}$ whereas funnel plot asymmetry was evaluated using Egger linear regression test with $\mathrm{P}<0.10$ significance level. A random-effects model (DerSimonian Laird) was used to calculate the pooled ORs in the overall and subgroup analyses. In order to account for potential imbalances in the baseline National Institute of health Stroke Scale (NIHSS) scores between the two groups given the non-randomized design of the majority of included studies, we conducted meta-regression. In this the dependent outcome was the effect size (log of the OR of the outcomes of interest, 3-month mortality, and 3 month FI), and the explanatory variables (independent variables) were baseline stroke severity quantified by the baseline average NIHSS score and the type of anesthesia (GA versus non-GA). ${ }^{30}$ We performed an equivalent $\mathrm{z}$ test for each pooled relative risk or hazard ratio, and two-tailed $P$ values of $<0.05$ were considered statistically significant. Statistical analyses were conducted using Review Manager (RevMan; computer program; version 5.3). Copenhagen: The Nordic Cochrane Center, The Cochrane Collaboration, 2014 and SPSS 16.0 (SPSS, Chicago, Illinois, USA).

\section{RESULTS}

\section{Study selection and characteristics}

Our search initially screened 2121 titles and abstracts from which 38 eligible studies were retained for full-text evaluation. After careful evaluation and no disagreements between the two reviewers, 21 studies were excluded (online supplementary table I) and 16 studies were selected that met the inclusion criteria, 1113 16-18 2123242628 31-33 35-37 (online supplementary figure I). One study ${ }^{38}$ was excluded because it was written in a language other than English.

We included a total of 16 studies comprising 5836 patients that compared GA with non-GA in patients with ELVO undergoing ET (table 1). Of all the included studies, nine studies included patients with AIS with anterior circulation occlusion, $131824262831-3335$ four studies included patients with both anterior and posterior circulation occlusions, ${ }^{11} 172336$ one study had only posterior circulation occlusion, ${ }^{16}$ and the location of vessel occlusion was not reported in two studies. ${ }^{21} 37$ The largest study had 2512 patients $(n=1999$, GA and $n=513$, non-GA), and the smallest study had 44 patients $(n=15$, GA and $n=29$, non-GA). Hypertension was present in $66.3 \%$ of patients in the GA group and $62.4 \%$ patients in the non-GA group. In the GA group, $33.3 \%$ were smokers compared with $30.2 \%$ of patients in the non-GA group. The prevalence of coronary artery disease(CAD) was noted in 25\% of GA group and $19.4 \%$ in non-GA group. Remaining baseline characteristics are described in online supplementary table II.

\section{Study quality and publication bias}

Owing to the inclusion of post hoc analyses of RCTs, there was a high risk of selection, performance, and detection biases. The majority of the included RCTs had a low risk of reporting bias, attrition bias, and other biases (online supplementary table III).

We used the Newcastle-Ottawa Scale to assess the risk of bias among the included cohort studies (online supplementary table IV). The risk of selection and comparability biases was considered low in all the studies. Outcome bias was moderate for studies that did not report the data on patients lost to follow-up, 
Table 1 Characteristics of the included studies

\begin{tabular}{|c|c|c|c|c|c|c|}
\hline $\begin{array}{l}\text { Author or trial, year of } \\
\text { publication }\end{array}$ & Country & Study design & $\begin{array}{l}\mathrm{GA} / \text { non-GA } \\
\text { patients }(\mathrm{n})\end{array}$ & $\begin{array}{l}\text { GA/non-GA anterior and/or } \\
\text { posterior circulation patients }(n)\end{array}$ & $\begin{array}{l}\text { Converted } \\
\text { from non-GA to } \\
\text { GA (n) }\end{array}$ & Type of endovascular treatment \\
\hline ANSTROKE, ${ }^{31} 2017$ & Sweden & Randomized & $45 / 45$ & 45/45 (Anterior) & $7 / 45(15.6 \%)$ & Stent retriever and aspiration \\
\hline Abou-Chebl, ${ }^{11} 2014$ & USA & Retrospective & $196 / 85$ & $\begin{array}{l}\text { 170/82 (Anterior) } \\
\text { 26/3 (Posterior) }\end{array}$ & NA & Stent retriever/aspiration \\
\hline Abou-Chebl, ${ }^{36} 2015$ & Multicenter & Post hoc - IMS III & $147 / 269$ & $\begin{array}{l}\text { 142/263 (Anterior) } \\
5 / 6 \text { (Posterior) }\end{array}$ & NA & Prestent retrieval/aspiration \\
\hline GOLIATH $_{,}^{32} 2018$ & Denmark & Randomized & $65 / 63$ & 65/63 (Anterior) & NA & Stent retriever and aspiration \\
\hline Gratz, $^{13} 2014$ & Switzerland & Prospective & $196 / 31$ & 196/31 (Anterior) & NA & Stent-retriever/aspiration \\
\hline HERMES, $^{35} 2018$ & Multicenter & Post-hoc - 7 RCTs & $153 / 456$ & 153/456 (Anterior) & NA & Stent retriever and aspiration \\
\hline Jadhav $_{,}{ }^{16} 2017$ & USA & Retrospective & $61 / 61$ & 61/61 (Posterior) & $8 / 61(13.1 \%)$ & Stent retrieval/aspiration \\
\hline Jagani, ${ }^{17} 2016$ & USA & Retrospective & $38 / 61$ & $\begin{array}{l}\text { 25/60 (Anterior) } \\
13 / 1 \text { (Posterior) }\end{array}$ & $1 / 62(1.6 \%)$ & Prestent retrieval/aspiration \\
\hline Janssen, ${ }^{18} 2016$ & Multicenter & Retrospective & $53 / 31$ & 53/31 (Anterior) & $0 / 31(0 \%)$ & Stent retriever/aspiration \\
\hline Just $_{1}{ }^{11} 2016$ & Canada & Retrospective & $42 / 67$ & NA & $1 / 68(1.5 \%)$ & Prestent retrieval/aspiration \\
\hline McDonald, ${ }^{37} 2015$ & USA & Retrospective & $1999 / 513$ & Not specified & NA & Prestent retrieval/aspiration \\
\hline Mundiyanapurath, ${ }^{23} 2015$ & Multicenter & Prospective & $29 / 15$ & $\begin{array}{l}\text { 23/13 (Anterior) } \\
6 / 2 \text { (Posterior) }\end{array}$ & $2 / 17(11.8 \%)$ & Stent retriever/aspiration \\
\hline SIESTA, ${ }^{33} 2016$ & Germany & Randomized & $73 / 77$ & 73/77 (Anterior) & $11 / 77(14.3 \%)$ & Stent retriever and aspiration \\
\hline Slezak, ${ }^{24} 2017$ & Switzerland & Retrospective & $266 / 135$ & 266/135 (Anterior) & $10 / 132(7.6 \%)$ & Stent retriever/aspiration \\
\hline van den Berg, ${ }^{26} 2015$ & Netherlands & $\begin{array}{l}\text { Retrospective - Pretrial } \\
\text { cohort of MR CLEAN }\end{array}$ & $70 / 278$ & 70/278 (Anterior) & $10 / 278(3.6 \%)$ & Prestent retrieval/aspiration \\
\hline Whalin, ${ }^{28} 2014$ & USA & Retrospective & $133 / 83$ & 133/83 (Anterior) & $12 / 83(14.5 \%)$ & Stent retriever/aspiration \\
\hline
\end{tabular}

GA, general anesthesia; IMS III, Interventional Management of Stroke III; NA, not available; non-GA, local or monitored anesthesia; RCT, randomized controlled trial.

or the follow-up period was inadequate. The overall Newcastle-Ottawa score was 108/117 (92\%), representing an overall high quality.
Funnel plot inspection did not disclose evidence of asymmetry in studies reporting the outcomes of functional independence (online supplementary figure II) and mortality (online

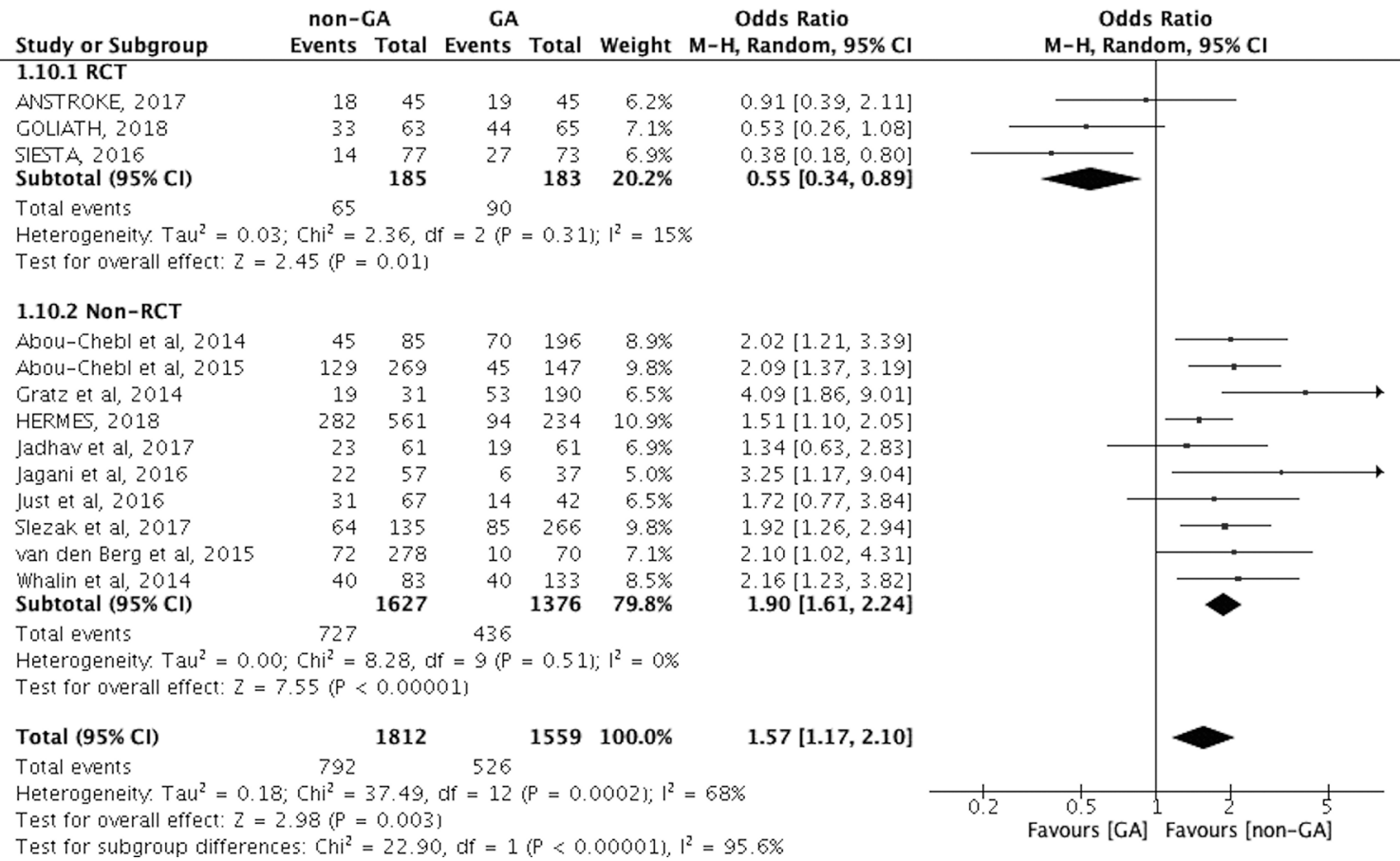

Figure 1 Forest plot showing the association of general anesthesia (GA), compared with local or monitored anesthesia (non-GA), with 3-month functional independence (defined as modified Rankin Scale scores of $0-2$ ) in all included studies stratified by study design (randomized vs nonrandomized). 


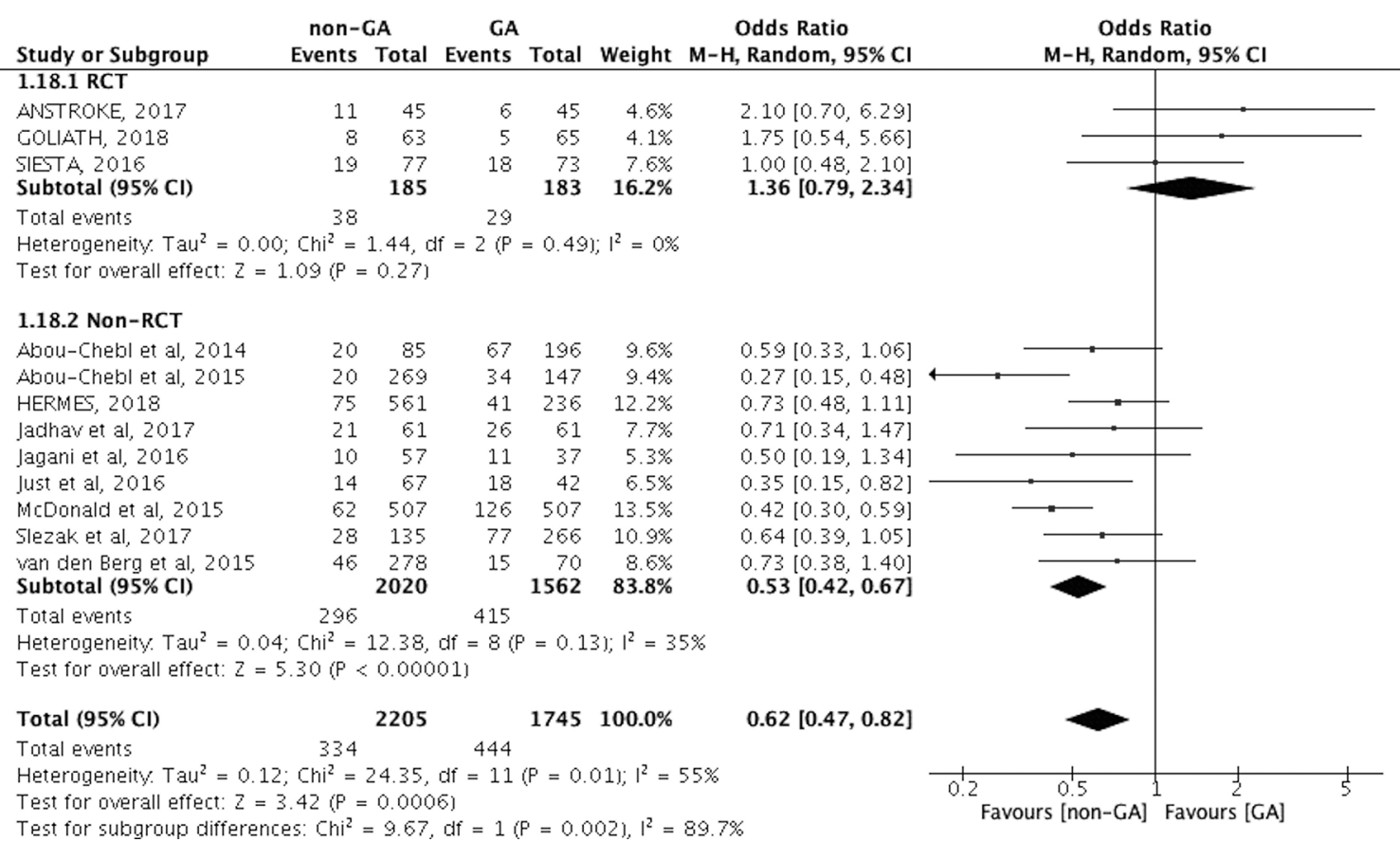

Figure 2 Forest plot showing the association of general anesthesia (GA), compared with local or monitored anesthesia (non-GA) , with 3-month mortality in all included studies stratified by study design (randomized vs non-randomized).

supplementary figure III). We also did not observe evidence of publication bias among studies reporting functional independence (Egger test, $\mathrm{P}$ value $=0.61$ ) and 3 -month mortality $(\mathrm{P}=0.85)$.

\section{Clinical outcomes}

In the pooled analysis of all included studies, non-GA was associated with a higher odds of 3 -month FI (13 studies; OR=1.57; 95\% CI 1.17 to $2.10 ; \mathrm{P}=0.003$; figure 1 and online supplementary figure IV), and a lower likelihood of 3 -month mortality (12 studies; $\mathrm{OR}=0.62 ; 95 \% \mathrm{CI} 0.47$ to $0.82 ; \mathrm{P}=0.0006$; figure 2 and online supplementary figure $\mathrm{V}$ ) and any ICH (11 studies; $\mathrm{OR}=0.83$; $95 \% \mathrm{CI} 0.69$ to $1.00 ; \mathrm{P}=0.05$; online supplementary figure VI). No significant difference was noted for sICH between the two groups (six studies; OR $=0.91 ; 95 \% \mathrm{CI} 0.60$ to $1.40 ; \mathrm{P}=0.68$; online supplementary figure VII). There was no evidence of heterogeneity for any $\mathrm{ICH}\left(\mathrm{I}^{2}=0 \%, \mathrm{P}\right.$ for Cochran $\mathrm{Q}$ statistic $=0.54)$ or $\operatorname{sICH}\left(\mathrm{I}^{2}=0 \%, \mathrm{P}\right.$ for Cochran Q statistic $\left.=0.65\right)$, whereas substantial heterogeneity was noted for functional independence $\left(\mathrm{I}^{2}=68 \%\right.$, P for Cochran $\mathrm{Q}$ statistic $\left.=0.0002\right)$ and mortality $\left(\mathrm{I}^{2}=55 \%, \mathrm{P}\right.$ for Cochran $\mathrm{Q}$ statistic $\left.=0.01\right)$.

\section{Procedural outcomes}

Effect on successful reperfusion, procedure time, bodily movements, and hemodynamic instability

The type of anesthesia was not associated with the odds of successful reperfusion (nine studies; $\mathrm{OR}=0.82 ; 95 \% \mathrm{CI} 0.59$ to $1.14 ; \mathrm{P}=0.25 ; \mathrm{I}^{2}=28 \%, \mathrm{P}$ for Cochran $\mathrm{Q}$ statistic $=0.20$; online supplementary figure VIII). Non-GA was associated with lower odds of hemodynamic instability (four studies; $\mathrm{OR}=0.33$; $95 \%$ CI 0.19 to $0.59 ; \mathrm{P}=0.0001 ; \mathrm{I}^{2}=0 \%, \mathrm{P}$ for Cochran $\mathrm{Q}$ statistic $=0.83$; online supplementary figure IX). GA was associated with a lower likelihood of bodily movements during ET (two studies; $\mathrm{OR}=0.04 ; 95 \% \mathrm{CI} 0.00$ to $0.28 ; \mathrm{P}=0.001$; online supplementary figure $\mathrm{X}$ ) without any evidence of heterogeneity $\left(\mathrm{I}^{2}=0 \%, \mathrm{P}\right.$ for Cochran $\mathrm{Q}$ statistic $\left.=0.60\right)$. Comparison of procedural time between GA and non-GA groups was evaluated (online supplementary table V). Mean procedural time ( \pm SD, minutes) from groin puncture to recanalization was $75.7 \pm 25.8$ for GA versus $81.3 \pm 32.3$ for the non-GA group ( $\mathrm{P}$ value $<0.001)$.

Effect on ventilator-associated and procedural complications

Non-GA was associated with a lower likelihood of respiratory complications (six studies; $\mathrm{OR}=0.59 ; 95 \% \mathrm{CI} 0.40$ to 0.87 ; $\mathrm{P}=0.007$; online supplementary figure $\mathrm{XI}$ ) without any evidence of heterogeneity across trials $\left(\mathrm{I}^{2}=35 \%\right.$, P for Cochran $\mathrm{Q}$ statistic $=0.17)$. No significant difference was observed for procedure-related complications between the groups (five studies; $\mathrm{OR}=0.79 ; 95 \% \mathrm{CI} 0.52$ to $1.21 ; \mathrm{P}=0.29$; online supplementary figure XII) without any evidence of heterogeneity $\left(\mathrm{I}^{2}=0 \%, \mathrm{P}\right.$ for Cochran Q statistic $=0.77$ ).

\section{Sensitivity analyses}

Additional analyses were conducted based on the study design (RCT vs observational studies), and type of endovascular device (pre-stentriever vs stentriever groups).

A. Non-GA was associated with a lower likelihood of 3-month FI (three RCTs; OR $=0.55 ; 95 \%$ CI 0.34 to $0.89 ; \mathrm{P}=0.01$; $\mathrm{I}^{2}=15 \%$, P for Cochran Q statistic $=0.31$; figure 1) in the RCT subgroup. However, no such association was noted for mortality (three RCTs; OR $=1.36$; $95 \%$ CI 0.79 to 2.34 ; $\mathrm{P}=0.27 ; \mathrm{I}^{2}=0 \%, \mathrm{P}$ for Cochran $\mathrm{Q}$ statistic $=0.49$; figure 2 ). Non-GA was related to higher likelihood of 3-month FI (10 studies; $\mathrm{OR}=1.90 ; 95 \% \mathrm{CI} 1.61$ to $2.24 ; \mathrm{P}<0.001 ; \mathrm{I}^{2}=0 \%$, $\mathrm{P}$ for Cochran Q statistic $=0.51$ ) and lower odds of mortality (nine studies; $\mathrm{OR}=0.53$; 95\% CI 0.42 to $0.67 ; \mathrm{P}<0.001$; $\mathrm{I}^{2}=35 \%, \mathrm{p}$ for Cochran Q statistic $=0.13$ ) in the subgroup of non-randomized studies.

B. Among studies published since 2015, use of pre-stentriever devices with non-GA was associated with a higher odds of 3 -month FI (four studies; OR $=2.12$; 95\% CI 1.54 to 2.90; $\mathrm{P}<0.001 ; \mathrm{I}^{2}=0 \%, \mathrm{P}$ for Cochran Q statistic $=0.82$; figure 3 ) and lower odds of mortality (five studies; $\mathrm{OR}=0.42 ; 95 \% \mathrm{CI}$ 


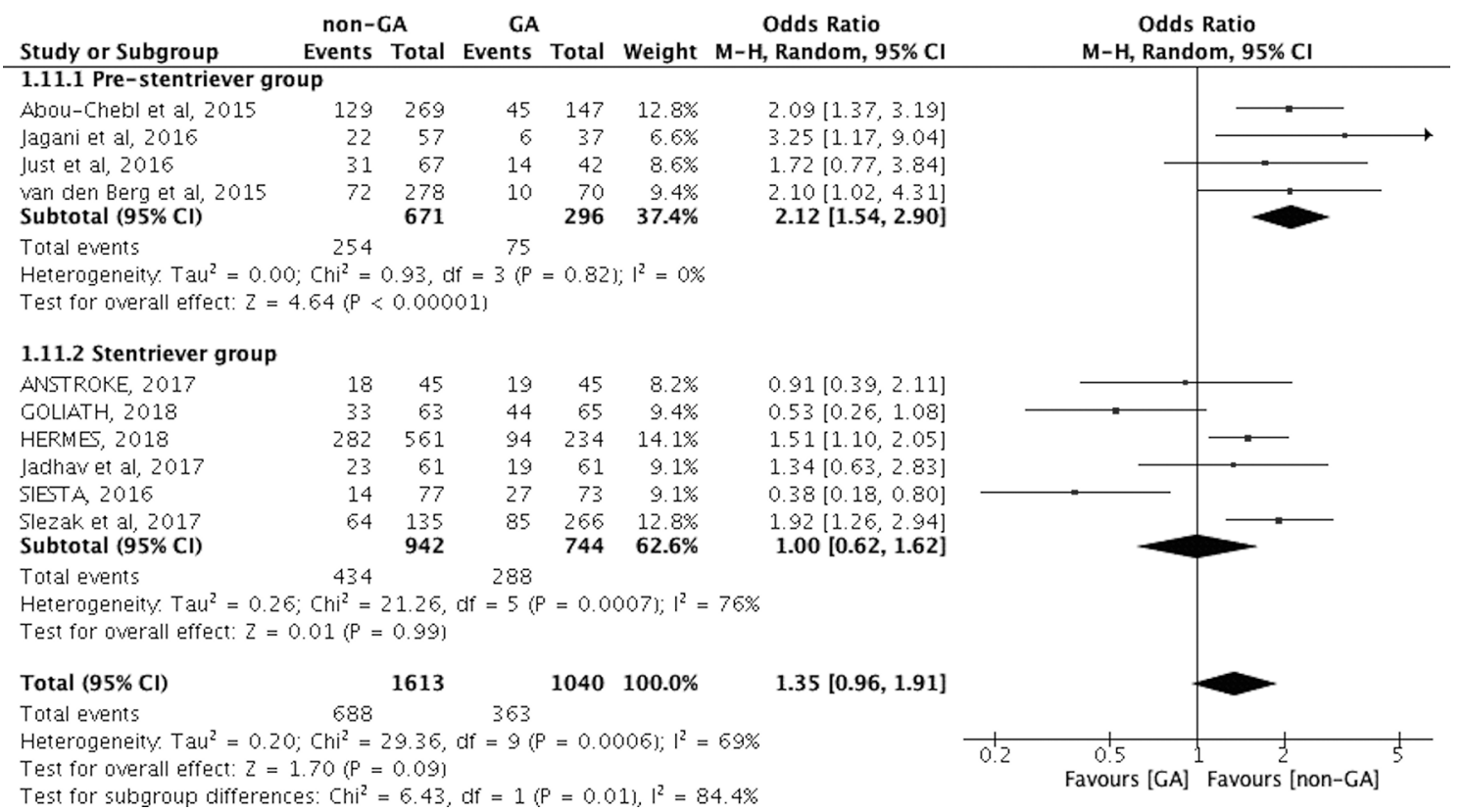

Figure 3 Forest plot showing the association of general anesthesia (GA), compared with local or monitored anesthesia (non-GA), with 3-month functional independence (defined as modified Rankin Scale scores of 0-2) in all included studies published since 2015 stratified by type of endovascular device (pre-stentriever/aspiration era vs stentriever/aspiration era).

0.31 to $0.57 ; \mathrm{P}<0.001 ; \mathrm{I}^{2}=24 \%$, P for Cochran Q statistic $=0.26$; figure 4$)$. On the contrary, no differences were observed, with the use of stentrievers, between the groups for 3-month FI (six studies; OR=1.00; 95\% CI 0.62 to $1.62 ; \mathrm{P}=0.99 ; \mathrm{I}^{2}=76 \%$, P for Cochran Q statistic $=0.0007$ ) or mortality (six studies; $\mathrm{OR}=0.83 ; 95 \% \mathrm{CI} 0.62$ to 1.12 ; $\mathrm{P}=0.23 ; \mathrm{I}^{2}=18 \%, \mathrm{P}$ for Cochran $\mathrm{Q}$ statistic $=0.30$ ).

\section{Subgroup analyses}

To assess the preference of type of anesthesia for patients with AIS with posterior circulation occlusions, we evaluated the studies including patients only with posterior circulation occlusions. Interestingly, there was a lower odds of preference for non-GA in patients with posterior circulation occlusions (four studies; $\mathrm{OR}=0.24 ; 95 \% \mathrm{CI} 0.07$ to $0.86 ; \mathrm{P}=0.03$ ); however, considerable heterogeneity was noted $\left(\mathrm{I}^{2}=61 \%, \mathrm{P}\right.$ for Cochran $\mathrm{Q}$ statistic $=0.05$; online supplementary figure XIII). Similarly, we evaluated the studies involving patients only with anterior circulation occlusions. No differences were observed between the two groups for 3-month FI (eight studies; OR=1.36; 95\% CI 0.87 to $2.12 ; \mathrm{P}=0.17 ; \mathrm{I}^{2}=78 \%, \mathrm{P}$ for Cochran $\mathrm{Q}$ statistic $<0.001$; online supplementary figure XIV) or mortality (seven

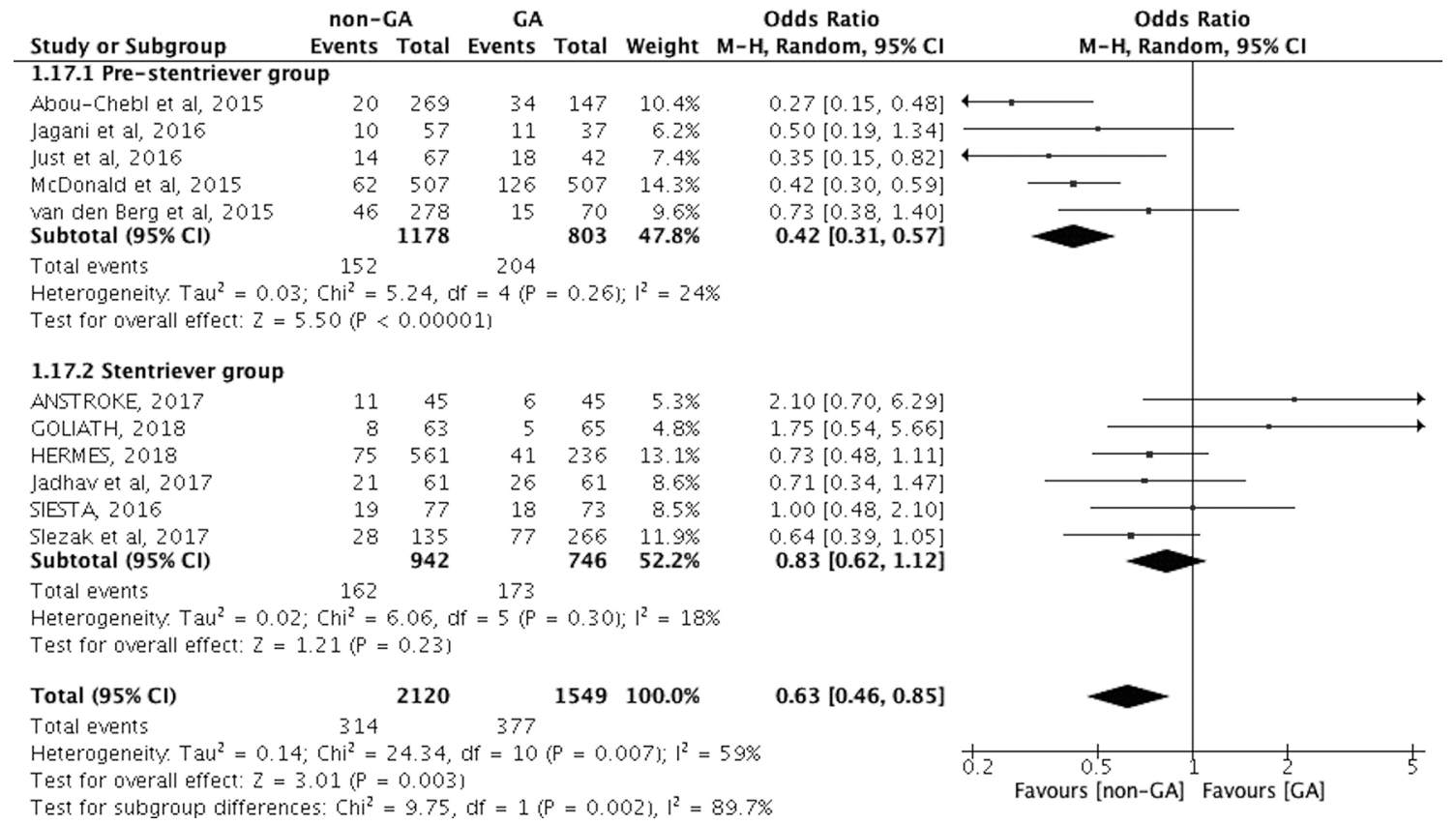

Figure 4 Forest plot showing the association of general anesthesia (GA), compared with non-GA, with 3-month mortality in all included studies published since 2015 stratified by type of endovascular device (pre-stentriever/aspiration era vs stentriever/aspiration era). 
studies; $\mathrm{OR}=0.76 ; 95 \% \mathrm{CI} 0.53$ to $1.09 ; \mathrm{P}=0.14 ; \mathrm{I}^{2}=61 \%, \mathrm{P}$ for Cochran Q statistic $=0.02$; online supplementary figure XV).

\section{Adjusted and meta-regression analyses}

We assessed all the studies that reported the adjusted outcome measures for both GA and non-GA. After adjusting for potential confounders, non-GA demonstrated a favorable profile for FI (three studies; $\mathrm{OR}=1.66 ; 95 \% \mathrm{CI} 1.22$ to $2.25 ; \mathrm{P}=0.001$; $\mathrm{I}^{2}=0 \%, \mathrm{P}$ for Cochran Q statistic $=0.90$; online supplementary figure XVI) and mortality (three studies; $\mathrm{OR}=0.47 ; 95 \% \mathrm{CI} 0.30$ to $0.73 ; \mathrm{P}=0.0007 ; \mathrm{I}^{2}=57 \%$, P for Cochran $\mathrm{Q}$ statistic $=0.10$; online supplementary figure XVII) in non-randomized studies reporting adjusted analyses.

We additionally performed meta-regression analyses after adjusting for baseline stroke severity. After adjusting for baseline NIHSS score, GA was not associated with the outcome of 3-month mortality $(\mathrm{OR}=1.15 ; 95 \% \mathrm{CI} 0.90$ to $1.48 ; \mathrm{P}=0.224$; online supplementary figure XVIII) and 3-month FI (OR $=0.91 ; 95 \% \mathrm{CI}$ 0.80 to $1.03 ; \mathrm{P}=0.118$; online supplementary figure $\mathrm{XIX}$ ).

\section{DISCUSSION}

Our updated meta-analysis shows that patients treated with non-GA during ET have a higher chance of 3-month FI and lower likelihood of any ICH, hemodynamic instability, respiratory complications and 3-month mortality. No significant differences were seen between the two options in the rates of sICH, successful reperfusion, and overall periprocedural complications. However, substantial heterogeneity was noted for the majority of reported associations across included studies and both unadjusted and adjusted analyses were probably skewed by non-randomized studies. Finally, the meta-analysis of three RCTs shows that non-GA is associated with lower odds of 3-month FI, while there is no relation between the type of anesthesia and mortality.

In this meta-analysis, we have highlighted the evidence for anesthetic options available for ET. Our findings partly corroborate the results of a recent meta-analysis, ${ }^{30}$ suggesting better outcomes in the non-GA subgroup. Brinjikji and colleagues reported higher odds of morbidity and mortality among patients undergoing GA. ${ }^{30}$ Although the authors did include recent RCTs, ${ }^{31-33}$ there was no stratification for the number of studies or type of assessment for various clinical outcomes. Furthermore, inclusion criteria of their study and ours are different. We primarily included studies that involved use of stent retrievers for ET (stentriever group) and studies that were published in 2015 and later, compared with meta-analyses done by Brinjikji et al, which included several studies from the pre-stentriever era published in $2010 .{ }^{30}$ Finally, our study reports additional outcome variables, such as hemodynamic instability, body movements and procedure time. Recent single-center RCTs and debates ${ }^{6}$ have highlighted the selection bias in previous non-randomized cohort studies. These usually reserved GA for patients with AIS with higher stroke severity or posterior circulation strokes. Our sensitivity analysis further highlights this selection bias of posterior circulation strokes among the GA group.

Several possible mechanisms have been proposed to explain the poorer outcomes associated with the use of GA. Apart from the attenuation of cerebral autoregulation, GA has been associated with rapid alterations in partial pressure of carbon dioxide, including either hypocapnia causing vasoconstriction and reduction of cerebral blood flow to penumbral tissue, or hypercapnia leading to vasodilatation and increased intracranial pressure. Similarly, cerebral hemodynamics is often affected by either excessive elevation or reduction of periprocedural MAP that could lead to worse clinical outcomes. ${ }^{12} 3139$ Commonly used general anesthetic agents, such as propofol and halogenated agents, ${ }^{4041}$ act as vasodilators that can compromise the cerebral blood flow. Davis and colleagues compared GA and non-GA groups and observed worse outcomes with lower blood pressures, especially with GA. ${ }^{12}$ However, prior retrospective studies lacked formalized study protocols or periprocedural specifications, whereas recent single-center RCTs diligently targeted the continuous monitoring and steady maintenance of a higher MAP during ET. ${ }^{31}$ Our study guides future trials to avoid anesthetic agents prone to cause wide swings in MAP or vasodilatation during anesthetic induction, and adopt protocols for continuous monitoring of hemodynamic parameters.

Various debates have discussed the delays involved in initiating ET, lack of concurrent clinical assessment, and ventilation complications when GA is used. ${ }^{2042}$ Of those, delay in procedural time secondary to mobilization of anesthesia, followed by evaluation and induction for endotracheal intubation were the major disadvantages. However, swift workflows were adopted in recent RCTs ${ }^{31-33}$ and demonstrated no statistical difference for image-to-groin puncture or image-to-recanalization times. Although an initial delay involved with induction for GA prolongs the image-to-groin puncture, better and safer working conditions during the procedure under GA may, in part, compensate by allowing a shorter groin puncture-to-recanalization time. Additionally, advancement and manipulation of ET devices under non-GA could lead to complications due to disrupted visualization of ET devices, dissection, perforation and subsequent hemorrhage. Concurrently, non-GA can require conversion to GA, which adds to the total procedure time, with resultant worse outcomes.

Similar to prior studies and RCTs, ${ }^{31-33}$ our meta-analysis confirms the higher odds of respiratory complications and pneumonia associated with GA. Patients with a higher NIHSS score, especially involving the posterior circulation, frequently manifest cardiorespiratory distress. In these clinical situations, the benefit of endotracheal intubation outweighs the risks associated with ventilator-related complications or pneumonias that are easily treatable with antibiotics. However, the timing of extubation becomes critical when using GA, especially for patients moving from the non-GA group secondary to extreme pain and/or agitation. Every effort should be made to extubate these patients immediately after the procedure to decrease the risk of hospital infections. Arguably, for situations where GA is necessary, swift workflows involving rapid pre-anesthetic induction, continuous physiological monitoring during the endovascular procedure, and early postprocedural extubation remain potential targets in future trials.

Certain limitations of this study need to be recognized. First, apart from a few RCTs and post hoc analyses, the majority of the included studies were non-randomized. Second, there was a selection bias towards a preference for GA for posterior circulation strokes, which inherently carry worse outcomes than anterior circulation strokes. To overcome these limitations, we performed sensitivity and subgroup analyses to demonstrate the 'true' effect of both GA and non-GA. Third, our outcomes were not stratified based on the location of vessel occlusion or lateralization based on cerebral hemisphere. Fourth, the documented associations between type of anesthesia and 3-month mortality and FI did not retain their significance in meta-regression analyses adjusting for baseline stroke severity. Thus, the documented imbalances in baseline NIHSS scores might have accounted for the initial associations of GA with worse clinical outcomes in the unadjusted analyses. Fifth, for some of the physiological outcome variables, such as hemodynamic instability and bodily movements, no uniform definitions were provided in the included 
studies. Finally, several potential explanations can be given for differences in outcomes seen with RCTs and non-RCTs. First, all the RCTs were single-center studies with small sample size, limiting the generalizability of their results. Second, and most importantly, all the RCTs adopted swift workflow and periprocedural study protocols especially for anesthesiologist availability and experience (trained in neurosurgical procedures), resulting in minimal delay in puncture time and low rate of anesthesia-related complications such as hypotension. On the other hand, non-RCTs reflect 'real-world' practice, where experienced anesthesiologists trained in neurosurgical procedures are not readily available for fast-paced stroke interventions. Lastly, the high conversion rate from non-GA to GA seen in two of the three RCTs might have contributed to time delay and outcome difference in the two groups. ${ }^{3133}$

In conclusion, our updated systematic review and meta-analysis demonstrates favorable results within the non-GA group, probably owing to the inclusion of non-randomized studies. Recent RCTs and post hoc analyses suggest either no difference in outcomes between the groups, or slightly better outcomes with the GA group. Additional multicenter RCTs are needed to demonstrate the positive effect of GA for patients with AIS undergoing ET.

Contributors NG, KM: Study concept and design, acquisition of data, analysis and interpretation, critical revision of the manuscript for important intellectual content. MFI: acquisition of data, critical revision of the manuscript for important intellectual content. GT, CN, DH, ASA, AVA, LE: critical revision of the manuscript for important intellectual content.

Funding The authors have not declared a specific grant for this research from any funding agency in the public, commercial or not-for-profit sectors.

Competing interests None declared.

Patient consent Not required.

Provenance and peer review Not commissioned; externally peer reviewed.

(c) Article author(s) (or their employer(s) unless otherwise stated in the text of the article) 2019. All rights reserved. No commercial use is permitted unless otherwise expressly granted.

\section{REFERENCES}

1 Goyal M, Demchuk AM, Menon BK, et al. Randomized assessment of rapid endovascular treatment of ischemic stroke. N Engl J Med 2015;372:1019-30.

2 Berkhemer OA, Fransen PS, Beumer D, et al. A randomized trial of intraarterial treatment for acute ischemic stroke. N Engl J Med 2015;372:11-20.

3 Campbell BC, Mitchell PJ, Kleinig TJ, et al. Endovascular therapy for ischemic stroke with perfusion-imaging selection. N Eng/ J Med 2015;372:1009-18.

4 Jovin TG, Chamorro A, Cobo E, et al. Thrombectomy within 8 hours after symptom onset in ischemic stroke. N Engl J Med 2015;372:2296-306.

5 Saver JL, Goyal M, Bonafe A, et al. Stent-retriever thrombectomy after intravenous t-PA vs. t-PA alone in stroke. N Engl J Med 2015;372:2285-95.

6 Rasmussen M, Simonsen CZ, Sharma D, et al. Letter by rasmussen et al regarding article, "anesthesia-related outcomes for endovascular stroke revascularization: a systematic review and meta-analysis". Stroke 2018;49:e20.

7 Hassan AE, Aman MM, Chauhdry SA, et al. Value of other endovascular techniques among patients with Merci device failure during the treatment of acute ischemic stroke: what to do when Merci fails? J Vasc Interv Neurol 2013:5:9-13.

8 McDonagh DL, Olson DM, Kalia JS, et al. Anesthesia and sedation practices among neurointerventionalists during acute ischemic stroke endovascular therapy. Front Neurol 2010;1:118.

9 Singer OC, Haring HP, Trenkler J, et al. Periprocedural aspects in mechanical recanalization for acute stroke: data from the ENDOSTROKE registry. Neuroradiology 2013:55:1143-51.

10 Abou-Chebl A, Lin R, Hussain MS, et al. Conscious sedation versus general anesthesia during endovascular therapy for acute anterior circulation stroke: preliminary results from a retrospective, multicenter study. Stroke 2010:41:1175-9.

11 Abou-Chebl A, Zaidat 00, Castonguay AC, et al. North American SOLITAIRE stent-retriever acute stroke registry: choice of anesthesia and outcomes. Stroke 2014;45:1396-401.

12 Davis MJ, Menon BK, Baghirzada LB, et al. Anesthetic management and outcome in patients during endovascular therapy for acute stroke. Anesthesiology 2012;116:396-405.
13 Gratz PP, Jung S, Schroth G, et al. Outcome of standard and high-risk patients with acute anterior circulation stroke after stent retriever thrombectomy. Stroke 2014;45:152-8.

14 Hassan AE, Chaudhry SA, Zacharatos H, et al. Increased rate of aspiration pneumonia and poor discharge outcome among acute ischemic stroke patients following intubation for endovascular treatment. Neurocrit Care 2012:16:246-50.

15 Hassan AE, Akbar U, Chaudhry SA, et al. Rate and prognosis of patients under conscious sedation requiring emergent intubation during neuroendovascular procedures. AJNR Am J Neuroradiol 2013;34:1375-9.

16 Jadhav AP, Bouslama M, Aghaebrahim A, et al. Monitored anesthesia care vs intubation for vertebrobasilar stroke endovascular therapy. JAMA Neurol 2017;74:704-9.

17 Jagani M, Brinjikji W, Rabinstein AA, et al. Hemodynamics during anesthesia for intraarterial therapy of acute ischemic stroke. J Neurointerv Surg 2016;8:883-8.

18 Janssen $H$, Buchholz G, Killer M, et al. General anesthesia versus conscious sedation in acute stroke treatment: the importance of head immobilization. Cardiovasc Intervent Radiol 2016;39:1239-44.

19 John S, Thebo U, Gomes J, et al. Intra-arterial therapy for acute ischemic stroke under general anesthesia versus monitored anesthesia care. Cerebrovasc Dis 2014:38:262-7.

20 Jumaa MA, Zhang F, Ruiz-Ares $G$, et al. Comparison of safety and clinical and radiographic outcomes in endovascular acute stroke therapy for proximal middle cerebral artery occlusion with intubation and general anesthesia versus the nonintubated state. Stroke 2010:41:1180-4.

21 Just C, Rizek P, Tryphonopoulos P, et al. Outcomes of general anesthesia and conscious sedation in endovascular treatment for stroke. Can I Neurol Sci 2016;43:655-8.

22 Li F, Deshaies EM, Singla A, et al. Impact of anesthesia on mortality during endovascular clot removal for acute ischemic stroke. J Neurosurg Anesthesiol 2014;26:286-90.

23 Mundiyanapurath S, Schönenberger S, Rosales ML, et al. Circulatory and respiratory parameters during acute endovascular stroke therapy in conscious sedation or general anesthesia. J Stroke Cerebrovasc Dis 2015;24:1244-9.

24 Slezak A, Kurmann R, Oppliger L, et al. Impact of anesthesia on the outcome of acute ischemic stroke after endovascular treatment with the solitaire stent retriever. AJNR Am J Neuroradiol 2017:38:1362-7.

25 Sugg RM, Jackson AS, Holloway W, et al. Is mechanical embolectomy performed in nonanesthetized patients effective? AJNR Am J Neuroradiol 2010;31:1533-5.

26 van den Berg LA, Koelman DL, Berkhemer OA, et al. Type of anesthesia and differences in clinical outcome after intra-arterial treatment for ischemic stroke. Stroke 2015;46:1257-62.

27 Werner M, Lopez-Rueda A, Zarco F, et al. Mechanical thrombectomy in acute basilar artery occlusion: a safety and efficacy single centre study. Interv Neuroradiol 2016:22:310-7.

28 Whalin MK, Lopian S, Wyatt K, et al. Dexmedetomidine: a safe alternative to general anesthesia for endovascular stroke treatment. J Neurointerv Surg 2014;6:270-5.

29 Brinjikji W, Murad MH, Rabinstein AA, et al. Conscious sedation versus general anesthesia during endovascular acute ischemic stroke treatment: a systematic review and meta-analysis. AJNR Am J Neuroradiol 2015;36:525-9.

30 Brinijkji W, Pasternak J, Murad MH, et al. Anesthesia-related outcomes for endovascular stroke revascularization: a systematic review and meta-analysis. Stroke 2017;48:2784-91.

31 Löwhagen Hendén P, Rentzos A, Karlsson JE, et al. General anesthesia versus conscious sedation for endovascular treatment of acute ischemic stroke: the Anstroke trial (Anesthesia During Stroke). Stroke 2017:48:1601-7.

32 Simonsen CZ, Yoo AJ, Sørensen LH, et al. Effect of general anesthesia and conscious sedation during endovascular therapy on infarct growth and clinical outcomes in acute ischemic stroke: a randomized clinical trial. JAMA Neurol 2018;75:470.

33 Schönenberger S, Uhlmann L, Hacke W, et al. Effect of conscious sedation vs general anesthesia on early neurological improvement among patients with ischemic stroke undergoing endovascular thrombectomy: a randomized clinical trial. JAMA 2016;316:1986-96

34 Sterne JA, Sutton AJ, loannidis JP, et al. Recommendations for examining and interpreting funnel plot asymmetry in meta-analyses of randomised controlled trials. BMJ 2011;343:d4002

35 Campbell BCV, van Zwam WH, Goyal M, et al. Effect of general anaesthesia on functional outcome in patients with anterior circulation ischaemic stroke having endovascular thrombectomy versus standard care: a meta-analysis of individual patient data. Lancet Neurol 2018:17:47-53.

36 Abou-Chebl A, Yeatts SD, Yan B, et al. Impact of general anesthesia on safety and outcomes in the endovascular arm of Interventional Management of Stroke (IMS) III Trial. Stroke 2015:46:2142-8.

37 McDonald JS, Brinjikji W, Rabinstein AA, et al. Conscious sedation versus general anaesthesia during mechanical thrombectomy for stroke: a propensity score analysis. J Neurointerv Surg 2015;7:789-94.

38 Langner S, Khaw AV, Fretwurst T, et al. [Endovascular treatment of acute ischemic stroke under conscious sedation compared to general anesthesia - safety, feasibility and clinical and radiological outcome]. Rofo 2013;185:320-7.

39 Froehler MT, Fifi JT, Majid A, et al. Anesthesia for endovascular treatment of acute ischemic stroke. Neurology 2012;79(13 Suppl 1):S167-73

40 Qureshi Al. Acute hypertensive response in patients with stroke: pathophysiology and management. Circulation 2008;118:176-87. 


\section{Ischemic Stroke}

41 Komatsu R, You J, Mascha EJ, et al. Anesthetic induction with etomidate, rather than propofol, is associated with increased 30-day mortality and cardiovascular morbidity after noncardiac surgery. Anesth Analg 2013;117:1329-37.
42 Gupta R. Local is better than general anesthesia during endovascular acute stroke interventions. Stroke 2010;41:2718-9. 